Proceedings of ASME Turbo Expo 2020

Turbomachinery Technical Conference and Exposition

GT2020

June 22-26, 2020, London, England

GT2020-14664

\title{
GAS TURBINE PERFORMANCE DIGITAL TWIN FOR REAL-TIME EMBEDDED SYSTEMS
}

\author{
Dr V. Panov ${ }^{1}$, Dr S. Cruz-Manzo² \\ 1Siemens Industrial Turbomachinery Ltd., Lincoln, UK \\ ${ }^{2}$ University of Lincoln, Lincoln, UK
}

\section{ABSTRACT}

This contribution reports on the development of Performance Digital Twin for industrial Small Gas Turbines. The objective of this study was the development of automation systems with control and monitoring functionalities, capable of addressing the requirements of future gas turbine plants for increased availability and reliability by use of Digital Twin technology.

The project explored development of Performance Digital Twin based on Real-Time Embedded computing, which can be leveraged with Internet-of-Things (IOT) Cloud Platforms. The proposed solution was provided in a form of modular software for a range of hardware platforms, with corresponding functionalities to support advanced control, monitoring, tracking and diagnostics strategies.

The developed Digital Twin was designed to be used in offline mode to assist the software commissioning process and in on-line mode to enable early detection of degradation and fault modes typical for gas path components. The Performance Digital Twin is based on a dynamic gas turbine model which was augmented with a Kalman tuner to enable performance tracking of physical assets.

To support heterogeneity of gas turbine Distributed Control Systems (DCS), this project explored deployment of Digital Twin on multiple platforms. In the paper, we discuss model-based design techniques and tools specific for continuous, discrete and hybrid systems.

The hybrid solution was deployed on PC-based platform and integrated with engine Distributed Control System in the field. Monitoring of gas turbine Performance Digital Twin functionalities has been established via Remote Monitoring System (STA-RMS). Assessment of deployed solution has been carried out and we present results from the field trial in this paper.

The discrete solution was deployed on a range of Programable Logical Controller (PLC) platforms and has been tested by integrating Digital Twin in virtual engine Distributed Control System network. The Performance Digital Twin was embedded in Single Master PLC and Master-Slave PLC configurations, and we present results from the system testing using virtual gas turbine assets. The IoT Platform MindSphere was integrated within virtual engine network, and in this contribution, we explore expansion of the developed system with Cloud based applications and services.

Keywords: Real-Time Embedded Systems, Digital Twins, Cyber-Physical Systems, Internet-of-Things

$\begin{array}{cl}\text { ACRONYMS } & \\ \text { CDP } & \text { Compressor Delivery Pressure } \\ \text { CDT } & \text { Compressor Delivery Temperature } \\ \text { CPS } & \text { Cyber-Physical System } \\ \text { cRSP } & \text { Common Remote Service Platform } \\ \text { DCS } & \text { Distributed Control System } \\ \text { DT } & \text { Digital Twin } \\ \text { GT } & \text { Gas Turbine } \\ \text { H-i-L } & \text { Hardware-in-the-Loop } \\ \text { HMI } & \text { Human Machine Interface } \\ \text { IDE } & \text { Integrated Development Environment } \\ \text { IoT } & \text { Internet of Things } \\ \text { RDC } & \text { Remote Diagnostic Center } \\ \text { RMS } & \text { Remote Monitoring System } \\ \text { RTM } & \text { Real-Time Model } \\ \text { PC } & \text { Personal Computer } \\ \text { PLC } & \text { Programable Logical Controller } \\ \text { SGT } & \text { Small Gas Turbine }\end{array}$

\section{INTRODUCTION}

The proliferation of the Digital Twin concept in industrial environments has led to the development of several variants of this technology applicable to almost the entire product life cycle $[1,2]$. In publication [2] following definition of Digital Twin is given: "A Digital Twin is defined as a virtual representation of a physical asset enabled through data and simulators for real-time prediction, monitoring, control and optimization of the asset for improved decision making through the life cycle of the asset and beyond". Traditionally we can recognize three main types of Digital Twins, namely: Product, Production and Performance Digital Twin. Product Digital Twin is used to support efficient 
design of new products by providing virtual-physical connection which enables performance analysis of complex multi-physics domains. Production Digital Twin supports optimized manufacturing process and enables more efficient production planning. Combining data generated from Product and Production Digital Twins, business can devise efficient production methodologies. Performance Digital Twins capitalize on data obtained from products in operation and makes this analysis available to activities such as asset management and operational optimization [3].

In this contribution we report on development of a Performance Digital Twin for an industrial Small Gas Turbine (SGT), which was closely integrated with plant automation systems [4]. The objective was creation of a functional CyberPhysical System (CPS) which would lead to improved availability and increased reliability of the product. The virtual gas turbine was created using a physics-based engine model deployed on a real-time platform, which was subsequently integrated with an engine Distributed Control System (DCS) [5]. The capability of the developed system was expanded with introduction of system connectivity to a Remote Monitoring System (STA-RMS) and the Internet-of-Things (IoT) platform (MindSphere).

\section{CPS AND IOT DOMAINS}

To achieve the objectives of flexible and robust operation of a Gas Turbine (GT) capable of adaptation to external as well internal conditions, this development explored integration of a self-configuring Virtual Gas Turbine with a physical turbine system. The hybrid CPS configuration containing a Virtual Gas Turbine generated from models of sensors, actuators and a gas turbine was integrated with the Physical Gas Turbine via deployment onto the engine DCS, which was extended with IoT connectivity [6]. In Fig.1. the overlapping nature of Physical, Cyber and IoT Domains is depicted [7].

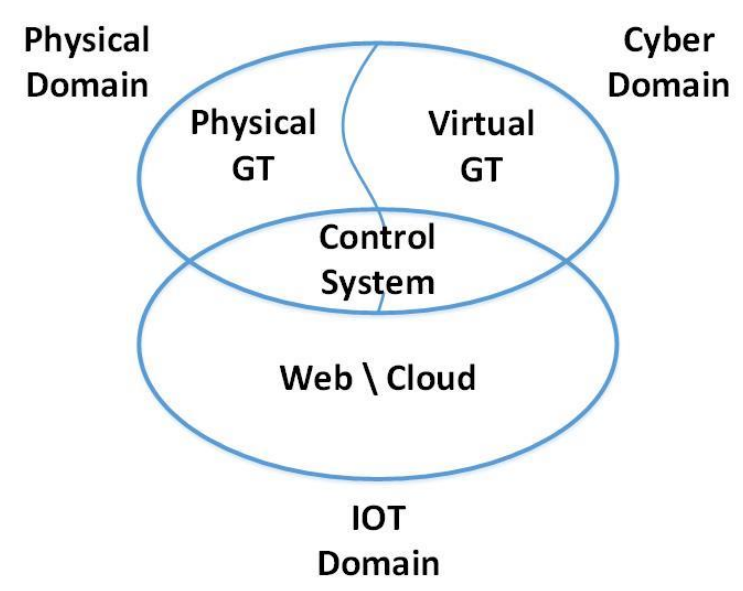

FIGURE 1: CPS AND IoT DOMAINS

The Physical Domain contains the real engine, i.e., gas turbine unit, which is connected to the automation system via multiple sensors and actuators. Human-in-the-loop interaction is also part of the Physical Domain, where the operator interacts through multiple modalities with this and other platforms of the system.

The self-configuring Virtual Gas Turbine, which is part of Cyber Domain, enables the Physical Gas Turbine to be monitored and controlled via adaptation to external operational and internal health conditions [8].

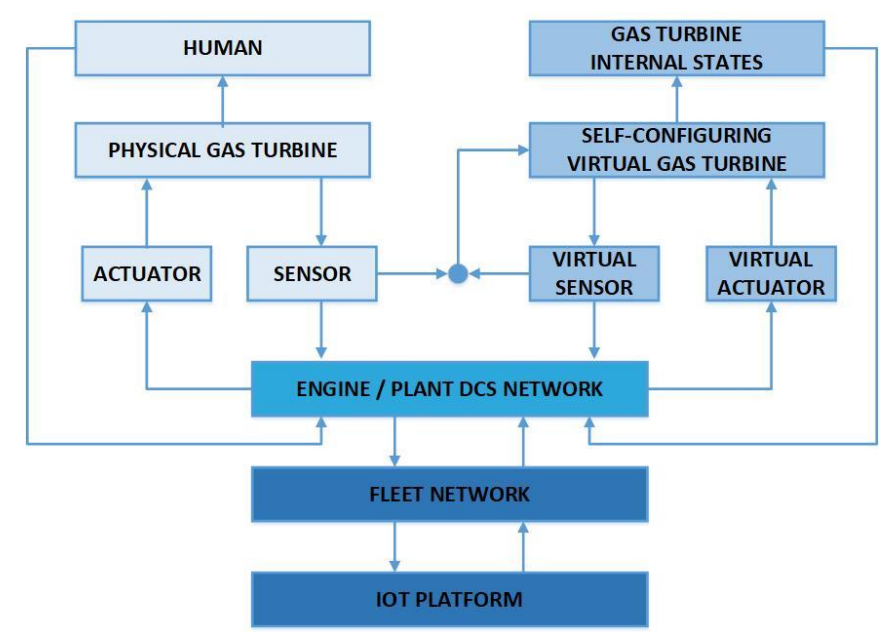

FIGURE 2: BUILDING BLOCKS OF CPS AND IoT DOMAINS

The IoT Domain contains state-of-the art network technologies based on standard and interoperable communication protocols [9]. The integrated IoT platforms provide a range of functionalities in support of MindSphere and STA-RMS applications. The physical engine trackable data objects (such as sensor measurements), and virtual engine smart data objects (such as internal engine states and virtual measurements), are seamlessly integrated into the information network.

The control system, which contains logical and communication components, is a subset of all three domains and serves as an integration platform for CPS and IoT Domains (Fig.2.).

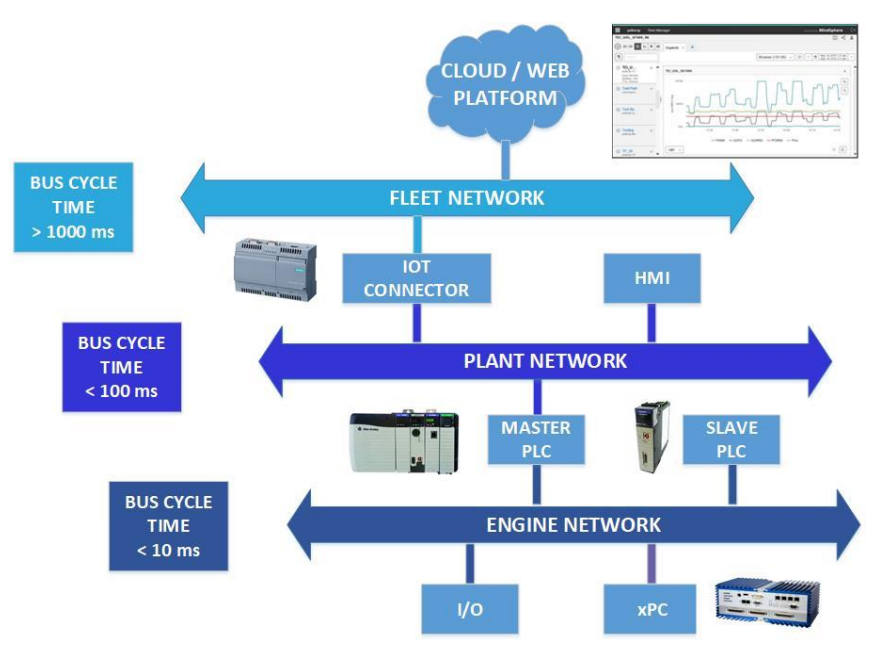

FIGURE 3: IOT PLATFORM CONNECIVITY 
The DCS supports integration of control and communication capabilities through a concept of multiple network levels. In the current integration set-up, there are three distinctive network levels: Engine network, Plant network and Fleet network (Fig.3.). This hierarchical network architecture enables deployment of heterogeneous computing platforms closely integrated with embedded systems. The state-of-the art DCS complies with demands for reliability, safety and security, and supports requirements related to synchronicity and trustworthiness of the deployed solution.

\section{DIGITAL TWIN ARCHITECTURE}

The core of the implemented Performance Digital Twin consists of several building blocks: RTM Predictor, Estimator, Kalman Tuner and Sensor Diagnostic blocks. The architecture of implemented Digital Twin is presented in figure Fig.4. The RTM Prediction block is obtained from a real-time physics-based nonlinear dynamic gas turbine model, which contains efficiency $(\eta)$ and flow capacity $(\Gamma)$ maps of major gas path components. The implemented component maps provide definition of predicted health parameters $(h)$, that correspond to nominal (new and clean) gas turbine engine. This Real-Time Model (RTM) is based on a system of ordinary differential equations and captures the dynamics of main gas paths components, rotating shafts, actuators and sensory elements [10]. The thermodynamic evaluation model is used for building the Estimator block. This block contains definitions for mass flow balance and thermodynamic and mechanical energy conservation [11]. The implemented gas turbine performance evaluation method contained within this block estimates gas turbine health parameters $(z)$ based on measurements from engine instrumentation $\left(y_{m}\right)$. Alongside available pressure and temperature measurements, devised evaluation method, also uses speed and acceleration / deceleration rates of rotating shafts in order to address dynamics of estimated health parameters.

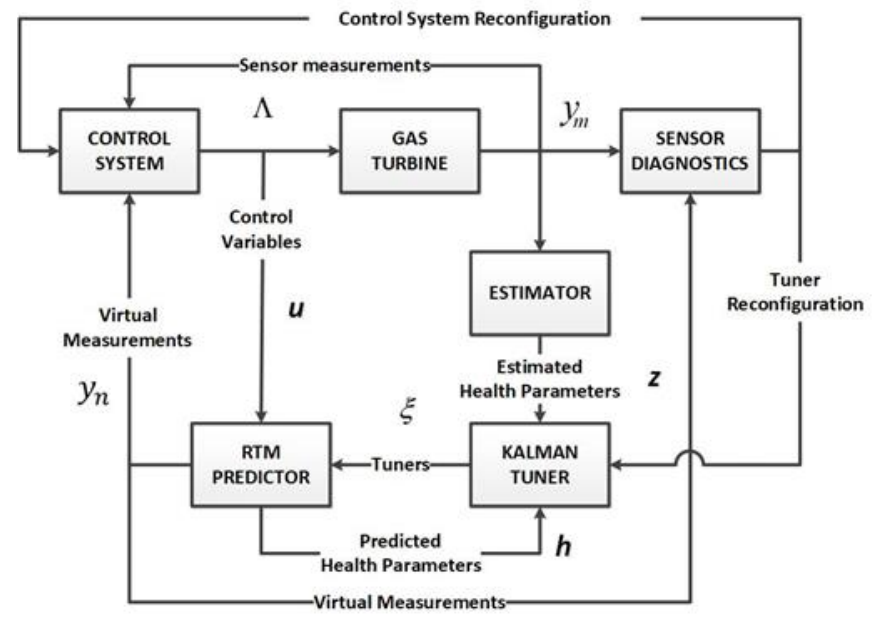

FIGURE 4: ARCHITECTURE OVERVIEW [13]

The auto-tuning process of the Digital Twin is contained within the Kalman Tuner block, where alignment of the Real-
Time Dynamic Model with the Physical Gas Turbine is achieved [12]. The structure of the Tuner block based on Kalman Filter is presented in Fig.5., where $w$ and $v$ represent zero mean Gaussian white noise, $K$ is Kalman Gain, and $k-1$ and $k$ represent discrete points in time. The implemented transition matrices are noted as $\Psi$ and $\Phi$, and they satisfy following conditions: $\Psi=$ $I-\Phi$ and $0 \leq \Phi \leq I$. In this formulation observation model noted with $H$ is represented with unit matrix $(H=I)$, considering that estimated health parameters are tuning model states, i.e. tuners directly. The Kalman Filter generates tuned health parameters $\xi$ based on smoothed health parameters evaluated by Estimator, i.e. estimated health parameters $z$.

The Sensor Diagnostics Block supports the Virtual Digital Twin self-configuring functionality [13]. Firstly, sensor diagnostic process takes place with subsequent isolation and accommodation of faulty sensors. Secondly, depending on severity of isolated faults, re-configuration of control system and tuning process is initiated, which allows self-configuration of the Digital Twin.

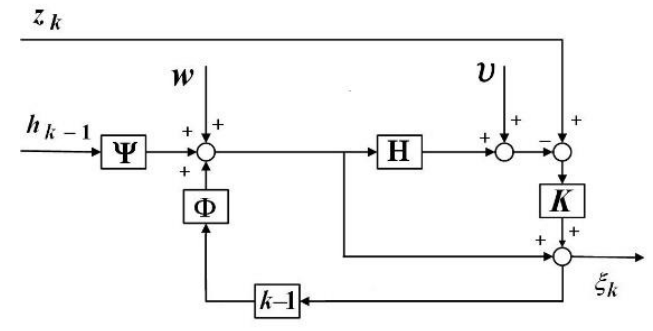

FIGURE 5: KALMAN FILTER STRUCTURE

The self-configuring functionality enables a myriad of possible configuration of the Virtual Gas Turbine depending on the number, location and severity of sensor faults. Two extreme configurations of the Digital Twin are presented in figures Fig.6. and Fig.7. In the case when all sensors are healthy, the Estimator is not affected and therefore the tuning process is fully preserved (Fig 6.).

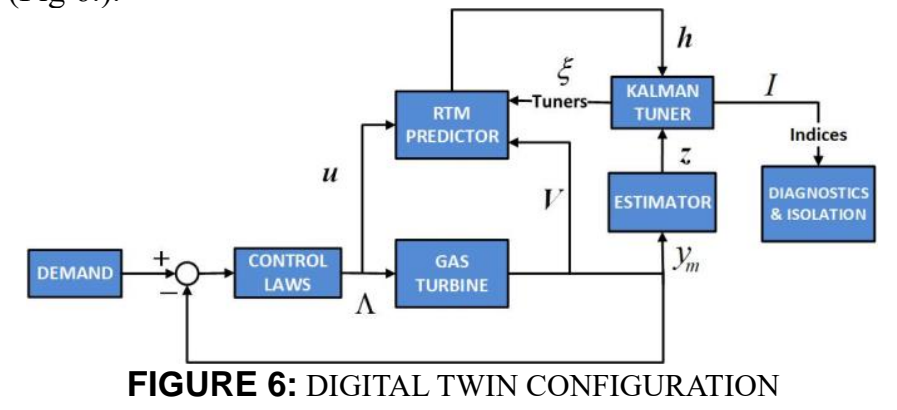

On the other side of configuration spectrum, when a combination of sensor faults removes confidence in the estimation process, adaptation of the dynamic model is not possible, and therefore alignment of the Digital Twin with the physical gas turbine is not active.

This computational process corresponds to simulator configuration (Fig. 7.). This configuration can be used in off-line 
mode to assist in the control software commissioning process, by following gas turbine schedule defined with predetermined load conditions $(V)$.

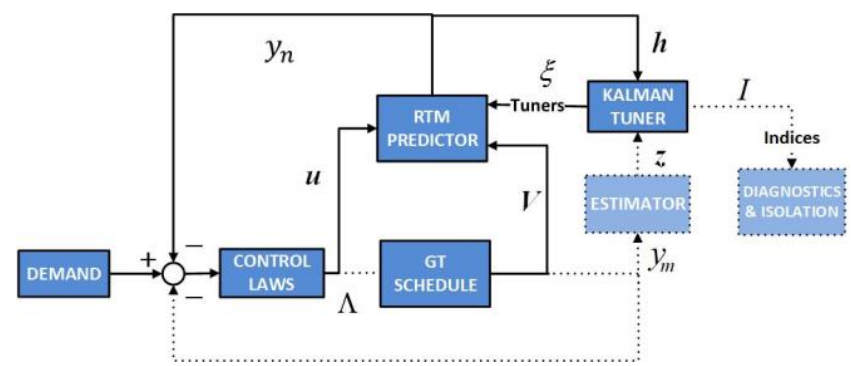

FIGURE 7: SIMULATOR CONFIGURATION

From a functionality perspective, development of the Digital Twin focused on five distinctive areas. Tracking of gas turbine performance capable of accounting for engine-to-engine variations and engine deterioration. Performance Diagnostics functionality based on health indices related to the main gas turbine components, and capable of capturing typical gas path degradation and fault modes [14]. Methods for estimation of remaining useful life of gas path components based on linear and non-linear regression modelling are contained within the Prognostics module. Soft sensors of the gas turbine Digital Twin are encapsulated with the Sensor Detection, Isolation and Accommodation module and they provide analytical redundancy for engine pressure and temperature measurements. The Performance Optimization module, which utilizes Model-Based Control strategies [15], was developed to support reduction of gas path related trips and to optimize engine performance in operation.

\section{CONTINUOUS AND DISCRETE SYSTEMS}

This project explored discrete and hybrid approaches for implementation of the developed Digital Twin through utilization of discrete and continuous simulation methods (Fig.8.).

Initially, a digital twin solution was developed as a continuous simulation system deployed onto PC based platform. This platform was running continuous simulation model deployed on industrial personal computer (PC) with real-time kernel system. The deployed solution was expanded with a realtime communication interface complying with Profibus field-bus protocol to enable integration with the gas turbine DCS. The implemented system is dependent on continuous time ( $t$ ) implementation of the Digital Twin prediction model which can be presented with the following generic system of equations:

$$
\begin{aligned}
& \dot{x}(t)=f(t, x(t), u(t), \xi(t)) \\
& y_{n}(t)=g(t, x(t), v(t), \xi(t))
\end{aligned}
$$

The above system of equations consists of state (Eq. 1) and output (Eq. 2) equations, where $x$ represents system state vector, $y_{n}$ is system sensor outputs vector, $\xi$ is tuner vector, $u$ and $v$ are control inputs and random sensor noise vectors respectively. The vector $u$ is a subset of vector $\Lambda$, which contains full set of controlling variables used in the real gas turbine engine.

This initial solution represents a hybrid system consisting of continuous PC-based Digital Twin platform integrated with a digital DCS.

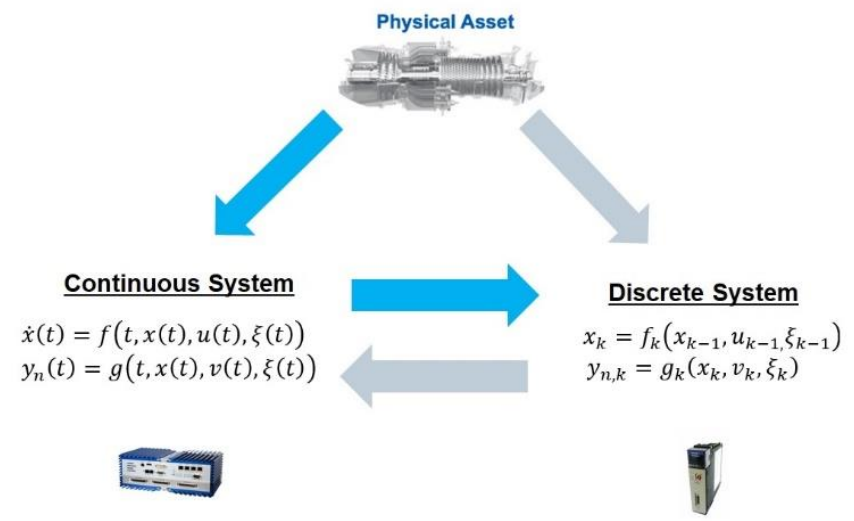

FIGURE 8: CONTINUOUS AND DISCRETE MODELLING AND SIMULATION

In follow-on implementation of the architecture, conversion of the continuous system into a discrete solution has been carried out to enable deployment of the Digital Twin solution onto PLC platforms. The continuous Digital Twin real-time dynamic model has been ported into a discrete model represented with following generic equations:

$$
\begin{aligned}
& x_{k}=f_{k}\left(x_{k-1}, u_{k-1}, \xi_{k-1}\right) \\
& y_{n, k}=g_{k}\left(x_{k}, v_{k}, \xi_{k}\right)
\end{aligned}
$$

where the above mathematical model describes system behavior at discrete points in time $k-1$ and $k$.

This iteration achieved a fully discrete solution and offered the possibility of deployment of the Performance Digital Twin into different DCS configurations. This project explored deployment onto two distinct configurations: Master and Slave PLC platforms. The implemented discrete-time solution differs from continuous-time implementation mainly in the type of engine model solvers used in corresponding deployments.

\section{SOFTWARE BUILD AND DEPLOYMENT}

The Performance Digital Twin has been created using Matlab \& Simulink toolchain and has been deployed onto $x P C$ Mobile Real-Time Target Machine (Fig.9.). Communication with the PLC platform has been established using Profibus communication protocol.

In this implementation the Simulink Real-Time tool was used to generate a stand-alone application based on a continuous model of the Digital Twin.

Further validation of system has been done by deploying the Digital Twin onto PLC based platforms used by SGT frame family. For this exercise, PLC Coder, a toolbox from the Matlab $\&$ Simulink toolchain, has been used for code generation and 
deployment, through utilization of compatible IDE platforms. PLC Coder generates hardware independent software blocks according to IEC 61131-3, the leading standard for PLC programming.

This project considered Simatic and Allen-Bradley PLC platforms used by the SGT product line. The code generated by PLC Coder from the discrete implementation of the Digital Twin, has been imported into third party Integrated Development Environments, STEP7 / TIA Portal and Studio5000 / RSLogix5000, which were used for code integration and deployment onto compatible PLC platforms.

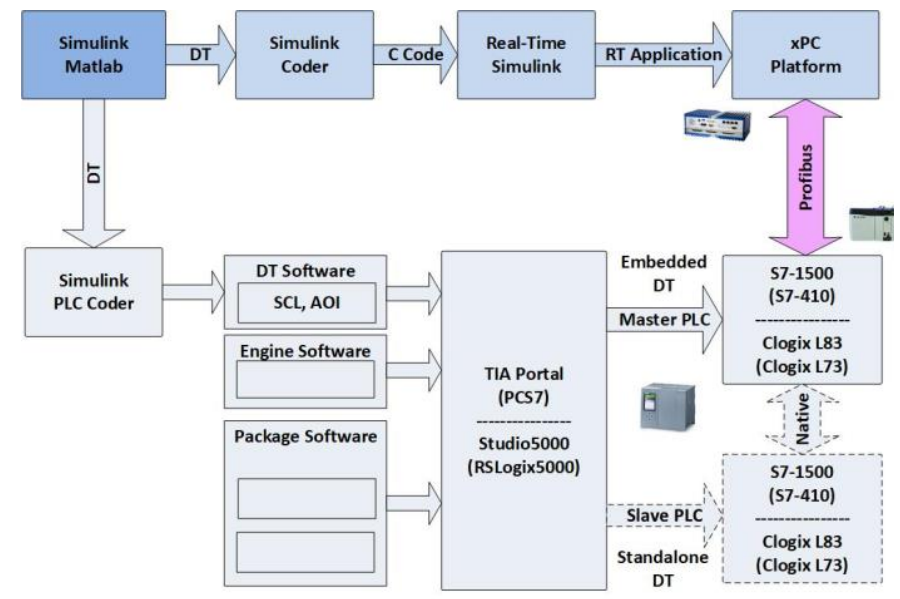

FIGURE 9: CODE GENERATION AND DEPLOYMENT

\section{DIGITAL TWIN - FIELD TRIAL}

A field trial of the Performance Digital Twin platform has been carried out at an operational site, where commissioning of the system has been completed during planned gas turbine outage. The SGT was used in a power generation application, and the Digital Twin has been integrated with the gas turbine DCS as a dedicated PC-based platform.

The deployed Digital Twin system has been created for Industrial Small Gas Turbine with following functionalities:

- Performance Tracking: tracking of gas turbine performance, based on auto-tuning of the engine model via a Kalman filtering process,

- Performance Diagnostics: generation of diagnostic data and monitoring of performance health indicators.

Real-time monitoring of the deployed platform has been established by remote connection with PC platform via secure common Remote Service Platform (cRSP) router. The on-line data have been visualized using an application deployed onto a remote Host PC.

The Performance Digital Twin has been deployed onto a PC platform which has been integrated with DCS system via Profibus Master DPV1 Communication module (Fig.10.). Diagnostic data generated by the Digital Twin platform are added to existing STA-RMS data link on a Human Machine Interface (HMI) platform and, as per standard connection configuration, the HMI machine was remotely connected via secure cRSP. The generated diagnostic data set has been made available to Remote Diagnostic Centre (RDC) for analysis in Remote Monitoring System (RMS).

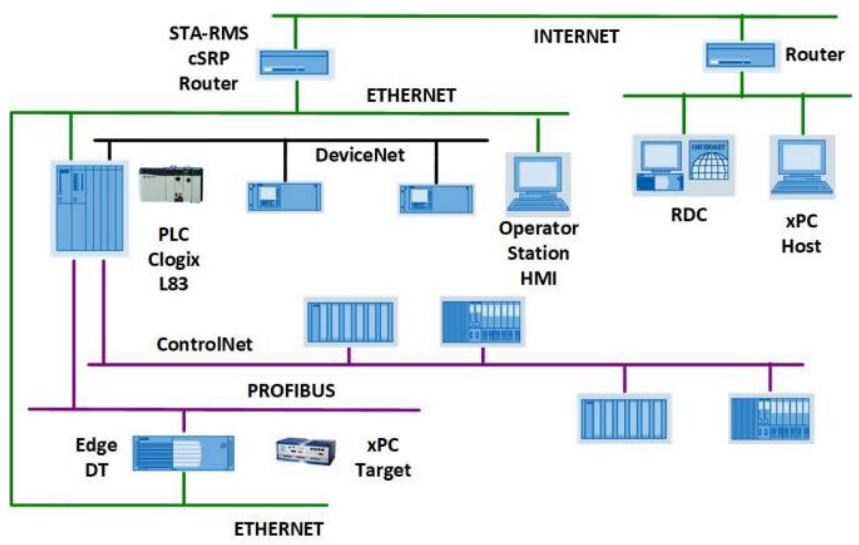

FIGURE 10: FIELD TRIAL PC BASED CONFIGURATION

The established remote connection with the PC platform supported the on-line monitoring of generated data sets, and also enabled remote configuration of the Digital Twin platform.

\section{Field Data - Compressor Fouling}

The data sets generated by the Digital Twin system deployed on the operational unit have been used for assessment of this field trial ( 7 months since PC platform commissioning). The Digital Twin system's ability to track engine operation, by aligning Real-Time Model (Predictor) using Estimator and Kalman Tuner functionalities, has been assessed. Examples of tracking of long-term trends and transient events are presented in this section.

The tracking process is based on a Kalman filtering technique, which aligns the real-time gas turbine model by adjusting health parameters for major gas path components. The residual deviations between predicted (nominal parameters for new and clean engine), and tuned health parameters (variations in component losses and flow capacity), are used to identify likely component degradation modes and faults. Usually this detection process is based on monitoring of "health indices" and tracking of long-term trends for health indicators generated by Digital Twin is presented. Health indices represent the percentage change in component characteristics, and typically two health indices can be defined for any component, which correspond to the capacity and efficiency index (Tab.1.).

TABLE 1: HEALTH INDICES

\begin{tabular}{|c|}
\hline Gas Path Component Capacity Index \\
\hline$I_{\Gamma}=100 \times \frac{\Gamma^{\xi}-\Gamma^{h}}{\Gamma^{h}}=100 \times \frac{\Delta \Gamma}{\Gamma^{h}}$ \\
\hline Gas Path Component Efficiency Index \\
\hline$I_{\eta}=100 \times \frac{\eta^{\xi}-\eta^{h}}{\eta^{h}}=100 \times \frac{\Delta \eta}{\eta^{h}}$ \\
\hline Superscripts: $\boldsymbol{h}$ - "Nominal" $/ \xi$ - "Tuned" \\
\hline
\end{tabular}


The operator utilized Small Gas Turbine (sub-15MW) across the load range, and engine has been operating on Temperature and Power limits.

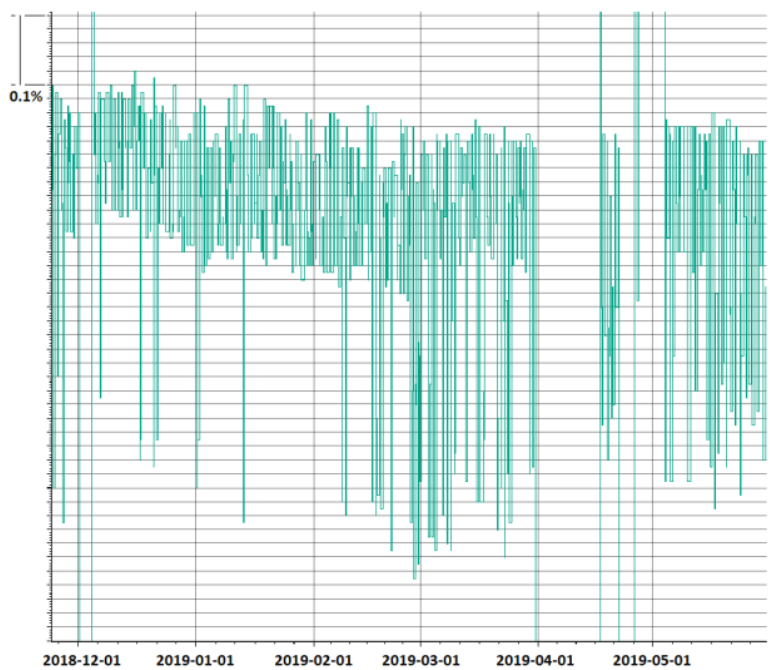

FIGURE 11: COMPRESSOR EFICIENCY INDEX

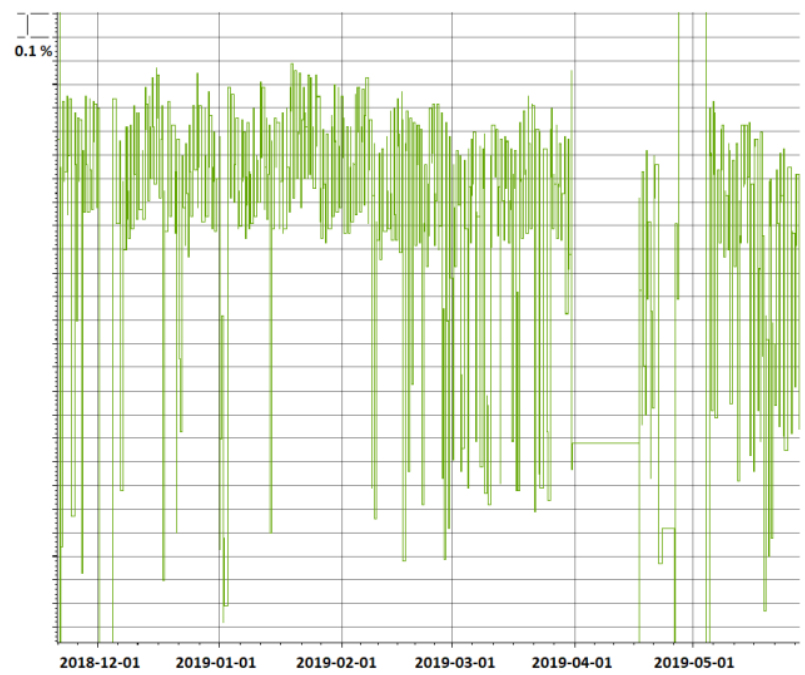

FIGURE 12: COMPRESSOR CAPACITY INDEX

Trends in calculated engine health indices is presented in figures Fig. 11. - Fig. 12. Health status of compressor component is presented with Compressor Efficiency (Fig.11.) and Compressor Capacity (Fig.12.) indices. In both trends one can observe reduction in health indices, which corresponds to compressor fouling, and agrees with observed trends in measured engine parameters [16].

This twin shaft gas turbine was operated as a power generation unit at full and part load, and corresponding variations in calculated health indices are captured in figures Fig.11. and Fig.12.
The ability of the Digital Twin to track transient events such as start-up, rundown and load change excursions, has been assessed by comparing engine measurements with Digital Twin analytical sensors "measurements". The comparison between engine and Digital Twin "measurements" of Compressor Delivery Temperature (CDT) and Compressor Delivery Pressure (CDP) is presented in figures Fig.13. and Fig.14. respectively.

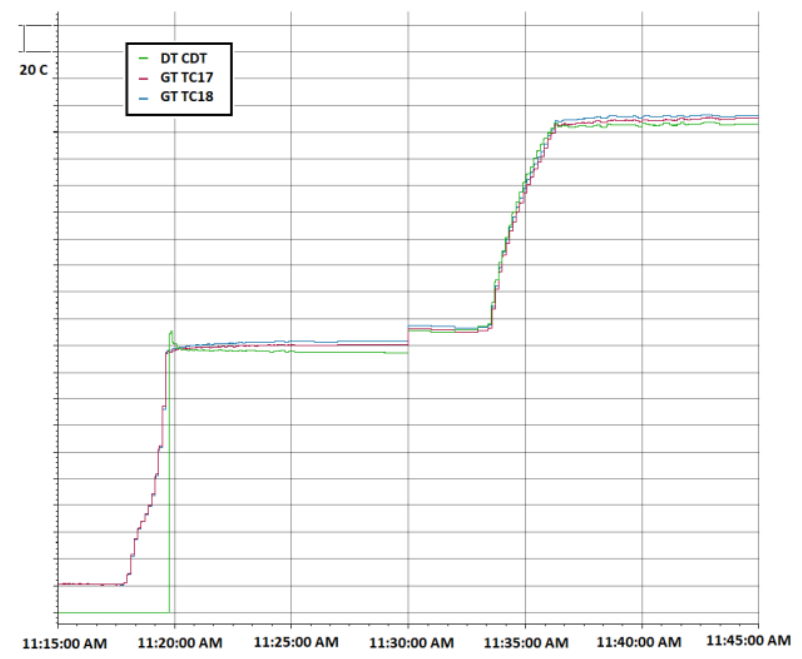

FIGURE 13: TRACKING OF CDT DURING START-UP

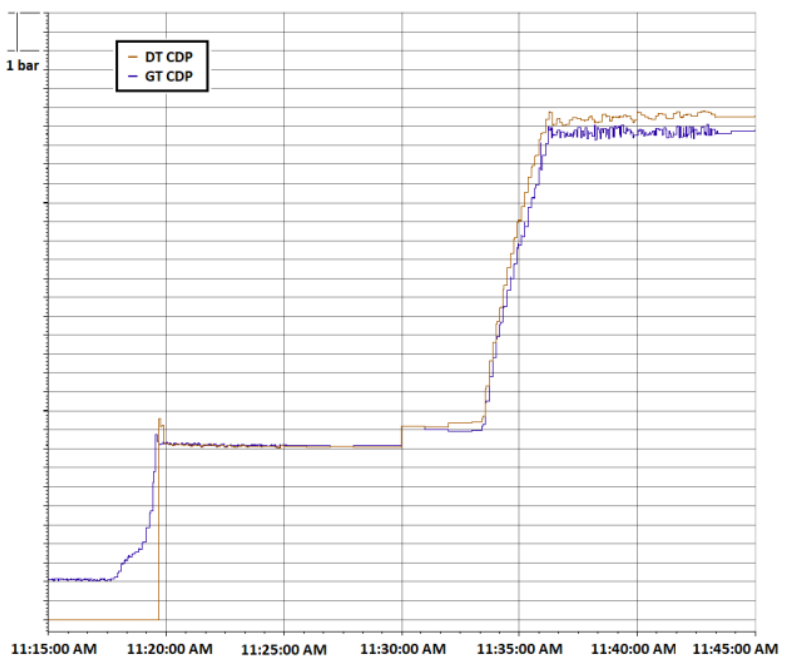

FIGURE 14: TRACKING OF CDP DURING START-UP

\section{DIGITAL TWIN - DCS FACILITY}

Extensive hardware-in-the-loop (H-i-L) trials have been carried out using SGT simulator facilities to assess the feasibility of Digital Twin deployment onto a range of PLC platforms (Fig. 15.). In this exercise connectivity of developed Digital Twin system with MindSphere IoT platform has been also demonstrated. 
Development of a real-time virtual system highlighted requirements related to application of novel hybrid discrete/continuous methods. In order to provide a sound foundation for robust design of an embedded Digital Twin, the model-based approach has been used in this contribution. Therefore, to support concept of tightly linked cyber and physical systems, high-fidelity virtual gas turbine model, deployable on continuous and discrete platforms, has been exploited.

Utilized gas turbine DCS concept with multi-level network architecture secured seamless integration of heterogenic platforms. Moreover, this concept offers the possibility of adaptability and scalability of Digital Twins in support of development of future Smart Plant systems.

Virtual and physical systems within CPS are closely integrated at multiple levels with network computing to provide physical systems with new capabilities. While this feature enables generation of new products and services due to ability to exchange vast information generated by Digital Twins connected to IoT platforms, this also increases risk of potential cyberattacks. Therefore, design process must consider data privacy and especially cybersecurity, because future active optimization functions of Digital Twin could have direct effect on the operation of the physical plant.

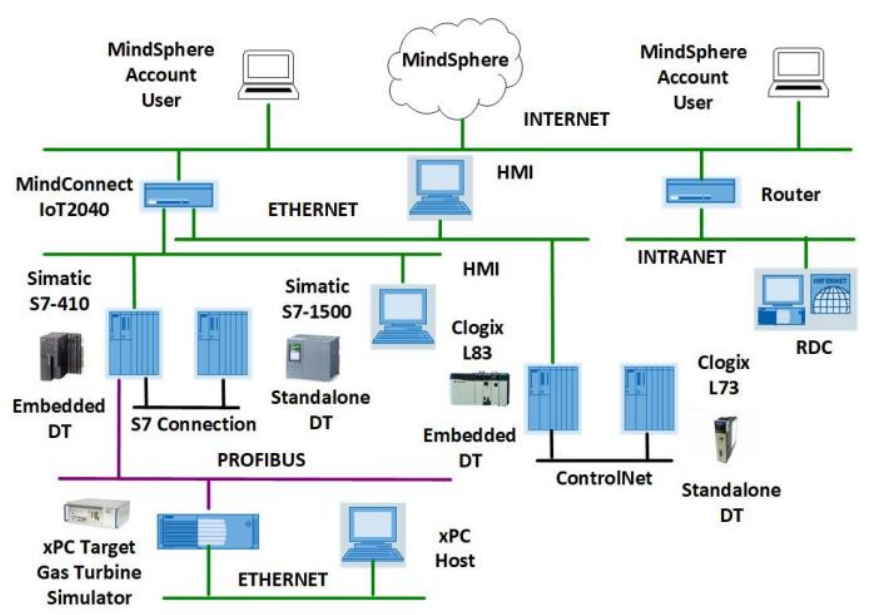

FIGURE 15: SIMULATOR FACILITY

This development raised question regarding optimal metrics for validation and certification of future versions of Digital Twin concepts, due to overall system complexity (real-time dynamism) and adaptability (self-configuration). Ever-evolving system functionalities, network interoperability and increasing software complexity impose challenges to system design requirements related to robustness, safety and security.

In this project we followed Evidence based validation and Modular certification concept. Validation of developed Digital Twin was carried out through operational experience on test beds and in field environment. On the other hand, the project used of- the-shelf hardware, and software modules were generated to be compliant with the IEC 61131-3 standard, which supports IEC Certification of fully integrated systems.

Depending on requirements related to real-time operation and needed processing power, Digital Twin functionalities can be distributed across different computational platforms. In this way system capabilities are expandable with non-real time functionalities which are encapsulated within Agents deployable on IoT platforms. This project demonstrated connectivity of developed Digital Twin with Remote Monitoring System (STARMS) and IoT platforms (MindSphere), which support hosting of Agents dedicated to fleet and asset data analytics.

\section{Degradation Simulation - PT Rubbing}

In order to test functionality of PLC implementation of Digital Twin, a simulation exercise was carried out using a gas turbine simulator integrated within virtual asset DCS.

The gas turbine simulator was used to simulate power turbine damage due to rotor tip rub [17]. The simulation test was performed for a twin shaft engine operating at part load condition, with subsequent injection of turbine efficiency reduction to simulate free turbine damage (Fig. 16.).

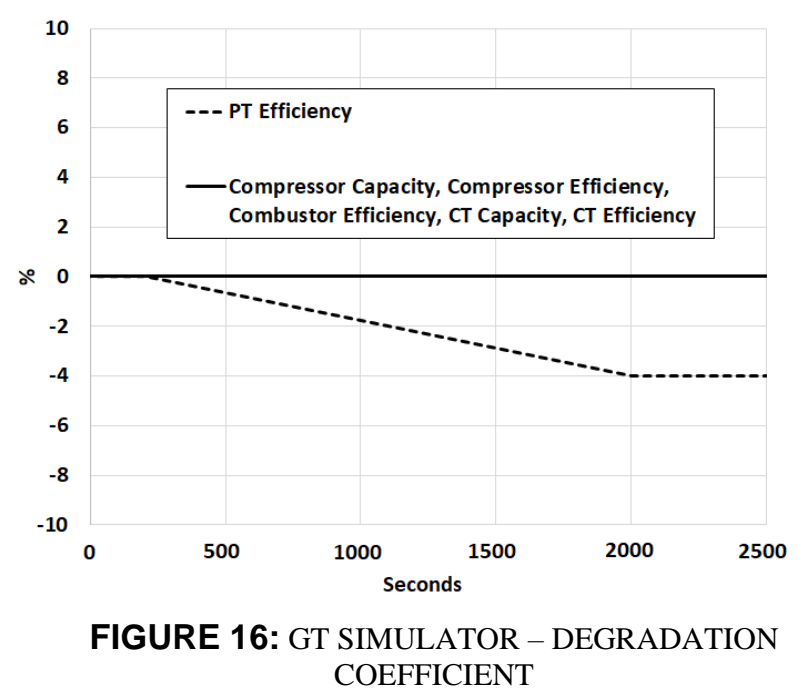

In this simulation exercise noise and bias of the engine sensors are implemented into the gas turbine simulator representing "real" engine to introduce model-plant mismatch.

The Digital Twin deployed onto PLC platform successfully resolved implanted levels of noise and bias for different engine instruments. The influence of the noise in the engine measurements is clearly visible as an increased level of noise in trends of the monitored health indices.

The results from this simulation case demonstrated the ability of the Digital Twin, deployed as a fully discrete solution, to track the injected fault by adjusting efficiency of the affected power turbine component (Fig.17.). 


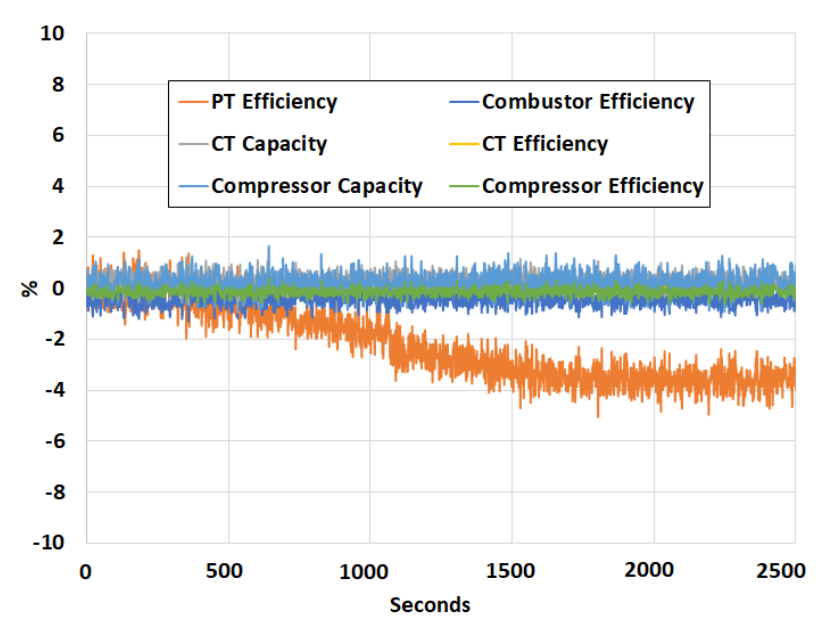

FIGURE 17: DT HEALTH INDICES

\section{CONCLUSION}

A field trial of the Performance Digital Twin system deployed onto a PC-based platform has been carried out at an operational site. Field data collected over a period of several months have been analyzed to assess performance of the deployed Digital Twin.

The Digital Twin system demonstrated an ability to achieve expected functionalities in terms of gas turbine performance tracking and diagnostics. Implemented Performance Digital Twin exploits multiple gas turbine sensors to identify deviations in estimated engine health states as indicators of typical gas path faults and degradation modes.

In the next field trial validation phase, the Digital Twin system will be deployed onto a PLC platform and integrated with the Gas Turbine DCS as a Standalone and Embedded implementation. Remote connectivity with the Digital Twin system will be established to demonstrate data transfer using STA-RMS and MindSphere platforms.

This emerging technology has ability to enhance current state-of -the-art Performance Digital Twin offerings which are predominantly based on non-real time and off-line solutions. The developed solution will facilitate the next generation of Gas Turbine Digital Twins, which will potentially exploit modular functionalities, distributed across the whole IoT chain consisting of Embedded, Edge and Cloud computational platforms.

\section{ACKNOWLEDGEMENTS}

This technology development was funded by Siemens Industrial Turbomachinery, Lincoln, U.K. The authors would like to thank School of Engineering at University of Lincoln for supporting this research.

\section{REFERENCES}

[1] Zhong, R. Y., Xu, X., Klotz, E., Newman, S. T., 2017, "Intelligent Manufacturing in the Context of Industry 4.0: A Review”, Engineering 3 (2017), 616-630.
[2] Rasheed, A., San, O., Kvamsdal, T., 2019, "Digital Twin: Values, Challenges and Enablers", arXiv:1910.01719.

[3] Zaccaria, V., Stenfelt, M., Aslanidou, I., Kyprianidis, K. G., 2018, "Fleet Monitoring and Diagnostics Based on Digital Twin of Aero-Engines", GT2018-76414, ASME Turbo Expo 2018, Oslo, Norway.

[4] Panov, V., 2016, "Integrated model-based control and health management for industrial gas turbine", PHM2016-041, 2016 IEEE International Conference on Prognostics and Health Management, Ottawa, Canada.

[5] Panov, V., 2011, "Distributed Network System for RealTime Model Based Control of Industrial Gas Turbines, GT2011-45298, ASME Turbo Expo 2011, Vancouver, Canada.

[6] Hehenberger, P., Vogel-Heuser, B., Bradley, D., Eynard, B., Tomiyama, T., Achiche, S., 2016, "Design, Modelling, Simulation and Integration of Cyber Physical Systems: Methods and Applications", Computers in Industry, Vol. 82, pp. 273-289.

[7] Green, C., Burns, M., Wollman, D., Griffor, E., 2019, "Cyber-Physical Systems and Internet of Things", NIST Special Publications 1900-202, 2019.

[8] Lee, E. A., Seshia, S. A., 2017, "Introduction to Embedded Systems - A Cyber-Physical System Approach", MIT Press, 2017.

[9] Oliver, S., Engber, M., Mich, R., Bohra, D., 2018, "Cloud Based Implementation of a Gas Turbine Remote Monitoring System", GPPS-2018-0149, GPPS Forum 18, Montreal, Canada.

[10]Panov, V., 2009, "GasTurboLib - Simulink Library for Gas Turbine Engine Modelling”, GT2009-59389, ASME Turbo Expo 2009, Orlando, USA.

[11] Panov, V., 2019, "Estimation of Health Parameters in Industrial Gas Turbines", US 10,254,752 B2, US Patent.

[12] Panov, V., 2014, "Auto-tuning of real-time dynamic gas turbine models", GT2014-25606, ASME Turbo Expo 2014, Dusseldorf, Germany.

[13] Panov, V., Maleki, S., 2017, "Model-Based Compensation of Sensor Failure in Industrial Gas Turbine", GPPF-2017$129,1^{\text {st }}$ Global Power and Propulsion Forum, Zurich, Switzerland.

[14]Panov, V., 2015, "Gas turbine performance diagnostics and fault isolation based on multidimensional complex health vector space", ETC2015-0051, 11 ${ }^{\text {th }}$ European Turbomachinery Conference, Madrid, Spain.

[15]Panov, V., 2013, "Model-based control and diagnostic techniques for operational improvement of gas turbine engines", ETC2013-0098, 10 $0^{\text {th }}$ European Turbomachinery Conference, Lappeenranta, Finland.

[16] Cruz-Manzo, S., Panov, V., Zhang, Y., 2018, "Gas Path Fault and Degradation Modelling in Twin-Shaft Gas Turbines", Machines 2018, 6(4), 43, doi.org/10.3390/machines6040043.

[17]Razak, A. M. Y., 2007, "Industrial Gas Turbines: Performance and Operability", Woodhead Publishing Limited, 2007. 